exploited the fact that the allosteric binding sites of the muscarinic receptors are expected to show more sequence diversity than the ACh binding sites.

The authors used five cell lines, each of which expressed a different member of the human muscarinic receptor family $\left(\mathrm{hM}_{1}-\mathrm{hM}_{5}\right)$. Application of LY2033298 in combination with ACh produced marked muscarinic receptor activation in the $\mathrm{hM}_{4}$ cell line, but not in any of the other cell lines. By administering LY2033298 to rats in conditioned avoidance responding and prepulse inhibition paradigms, the authors found preliminary evidence that this agent has an antipsychotic effect.

Chan et al. speculate that LY2033298 could be the first of a new class of muscarinic antipsychotic therapies that modulate neurotransmission without directly targeting dopamine or 5-HT receptors.

Original article Chan WY et al. (2008) Allosteric modulation of the muscarinic $\mathrm{M}_{4}$ receptor as an approach to treating schizophrenia. Proc Natl Acad Sci USA 105: 10978-10983

\section{Response to antidepressants is linked to TrkB signaling in forebrain progenitor neurons}

Scientists in the US have come closer to understanding why antidepressants do not always work - a poor treatment response could be related to impairment of TrkB-dependent neurogenesis in the forebrain.

More than a third of patients with depression do not respond to first-line treatment with current antidepressants. Previous research has indicated that neurogenesis might be a key requirement for these drugs to be effective; furthermore, brain-derived neurotrophic factor (BDNF) has been suggested to have a role in mediating the response to chronic antidepressant therapy. Research by Li et al. now shows that a functional link exists between these two mechanisms. In mice, ablation of TrkB (the gene that encodes a receptor for BDNF) in hippocampal neurogenic progenitor cells resulted in impaired neurogenesis and prevented the behavioral improvements associated with chronic antidepressant therapy that were seen in wild-type animals. Of note, deletion of TrkB in fully differentiated neurons of the same brain regions had no such effects.

Voluntary exercise can have effects on neurogenesis and behavior that are similar to those observed with chronic antidepressant therapy; therefore, Li et al. conducted a control experiment that studied the effects of TrkB deletion on the biological and behavioral responses to wheel running. The results confirmed the findings above. Further in vivo and in vitro observations suggested that reduced neurogenesis in the absence of TrkB is caused by a decrease in BDNF-induced proliferation of hippocampal precursor neurons.

Luis Parada, senior author of the study, concludes that "in a genetic model system, hippocampal neurogenesis is required to mediate chronic antidepressive effects. This may help explain why antidepressants only have beneficial effects after chronic treatment despite their immediate pharmacological activity upon consumption."

Original article Li Y et al. (2008) TrkB regulates hippocampal neurogenesis and governs sensitivity to antidepressive treatment. Neuron 59: 399-412

\section{Is antifibrinolytic therapy beneficial after aneurysmal subarachnoid hemorrhage?}

The $24 \mathrm{~h}$ rebleeding rate after aneurysmal subarachnoid hemorrhage is $4-20 \%$. Antifibrinolytic therapy reduces this rate, but the treatment has been associated with an increase in ischemic deficits. Some recent studies have shown, however, that acute antifibrinolytic therapy can decrease rebleeding without causing serious adverse effects, and a new study from the US has produced further evidence to support this view.

In this prospective study, Starke et al. investigated the effects of administration of $\varepsilon$ aminocaproic acid (EACA) for a maximum of $72 \mathrm{~h}$ from the time of diagnosis of subarachnoid hemorrhage. Of 248 enrolled patients, 73 received EACA and 175 did not. Rebleeding occurred in $2.7 \%$ of EACA-treated patients compared with $11.4 \%$ of the non-EACA group $(P=0.02)$, and a nonsignificant $76 \%$ relative reduction in rebleeding-related mortality was evident in the EACA-treated group. The incidence of ischemic deficit was similar in 